\title{
EGFR Tyrosine Kinase Inhibitor Therapy
}

National Cancer Institute

\section{Source}

National Cancer Institute. EGFR Tyrosine Kinase Inhibitor Therapy. NCI Thesaurus. Code C116534.

The use of EGFR tyrosine kinase inhibitors to disrupt the activation of EGFR, effectively halting the signaling cascade for cell growth, thereby decreasing tumor proliferation and migration. 